\title{
Brillouin optical time-domain analyzer for extended sensing range using probe dithering and cyclic coding
}

\author{
Haritz Iribas $^{\mathrm{a}}$, Alayn Loayssa ${ }^{\mathrm{a}}$, Florian Sauser ${ }^{\mathrm{b}}$, Miguel Llera $^{\mathrm{b}}$, and Sébastien Le Floch ${ }^{\mathrm{b}}$ \\ a Universidad Pública de Navarra, Campus Arrosadia s/n, 31006 Iruñea, Spain \\ ${ }^{\mathrm{b}} \mathrm{HE}-\mathrm{ARC}$, Rue de la Serre 7, 2610 Saint Imier, Switzerland
}

\begin{abstract}
We present an enhanced performance Brillouin optical time-domain analysis sensor that uses dual probes waves with optical frequency modulation and cyclic coding. The frequency modulation serves to increase the probe power that can be injected in the fiber before the onset of non-local effects and noise generated by spontaneous Brillouin scattering. This leads to higher detected signal-to-noise ratio (SNR), which is further increased by the coding gain. The enhanced SNR translates to extended range for the sensor, with experiments demonstrating 1-m spatial resolution over a $164 \mathrm{~km}$ fiber loop with a $3-\mathrm{MHz}$ Brillouin frequency shift measurement precision at the worst contrast position. In addition, we introduce a study of the power limits that can be injected in the fiber with cyclic coding before the appearance of distortions in the decoded signal.
\end{abstract}

Keywords: Brillouin distributed sensors, Brillouin optical time domain analysis, Cyclic coding

\section{INTRODUCTION}

Distributed optical fiber sensors based on Brillouin optical time-domain analysis (BOTDA) have been widely employed in civil and geotechnical engineering. For instance, in the monitoring of oil or gas pipelines, railway inspection, fire detection, etc... Many of these structures require a large sensing range. However, the sensing range of BOTDA sensors is limited by the attenuation of the fiber and by the maximum pump and probe power that can be injected in the fiber before the appearance of non-liner effects, non-local effects ${ }^{1,2}$ and noise induced by spontaneous Brillouin scattering. ${ }^{3}$ A number of techniques have been proposed to overcome these limitations, and achieve an improvement of the signal-to-noise ratio (SNR) of BOTDA sensors. For instance, the use of different coding techniques ${ }^{4,5}$ or distributed pump amplification. ${ }^{6}$

We have recently presented a dual-probe BOTDA in which the optical frequency of the probe waves is modulated in order to mitigate second-order non local effects and increase the Brilloiun threshold of the fiber link. ${ }^{7}$ In this work, we demonstrate that cyclic coding can be deployed in this setup in order to increase the detected SNR and hence the sensing range. Moreover, we introduce a first study on the power limits that can be injected in the fiber using mono-color cyclic coding in order to avoid distortion of the decoded signal.

\section{FUNDAMENTALS OF THE TECHNIQUE}

The fundamentals of the proposed sensor are schematically described in Fig. 1, where the optical waves involved in the technique are depicted. As it is highlighted in the figure, the optical frequency of the probe waves is modulated following a saw-tooth shape. ${ }^{7}$ Additionally, this frequency modulation (FM) is synchronized to the pump pulses so that a sequence of pulses experiences the same wavelength of the probe waves at any given location. The FM increases the Brillouin threshold limit of the fiber. Moreover, second order nonlocal effects are prevented because the FM modulation leads to a broadening of the effective gain and loss spectra induced by both probes on the pump, which mutually compensate avoiding any distortion of the pulse spectrum. Therefore, a very large probe power can be deployed to enhance the SNR in detection.

A.L.: E-mail: alayn.loayssa@unavarra.es

S.L.F.: E-mail: sebastien.lefloch@he-arc.ch 


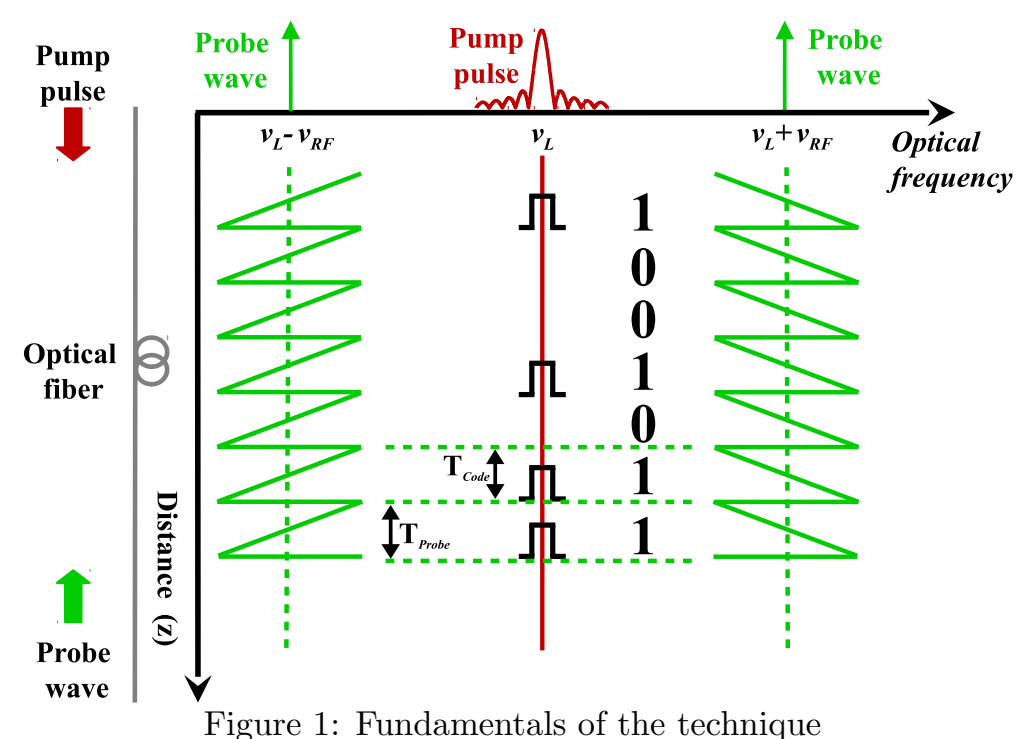

In order to further increase the SNR, it is interesting to combine the FM technique with mono-color cyclic coding. For this purpose, we introduce a code based on a circulant matrix of dimension L, with L a prime number. ${ }^{4}$ The code is generated following the recurrence equation for an integer $n$ :

$$
\left\{\begin{array}{c}
u_{1}=0 \\
u_{n+1}=\left(u_{n}+n\right) \bmod L
\end{array}\right.
$$

where mod is the modulo function and $n \in\left[1, \frac{L+1}{2}\right]$. The first line of the code matrix for a code length of seven is represented in Fig. 1. The improvement of the SNR given by the coding gain is $(L+1) / 2 \sqrt{L}$. It is important to point out that in order to decode properly the signal, the period of the frequency modulated probe waves must be equal to the distance between two of the contiguous bits of the generated cyclic code, $T_{\text {Code }}=T_{\text {Probe }}$.

In order to maintain an error-free decoding process for mono-color cyclic coding techniques, there is a limitation regarding the maximum pump and probe power that can be injected in the fiber. Firstly, in order to keep a linear Brillouin amplification of the probes, the total Brillouin gain induced by the pump pulses sequence on the probe needs to be relatively small. Thus, it is necessary to limit the pump power to avoid distortion. The linearity is actually one of the fundamental requirements to apply coding techniques. Moreover, as the successive pulses interact with a probe wave that has been amplified by previous pulses, non-local effects will appear earlier, which leads to the need to reduce the probe power. Finally, most of coding techniques require that all coded pump pulses have the same power, which limits the pump power due to the fact that the transient behavior of erbium-doped fiber amplifiers (EDFA) worsens for large power. All these limitations imply that cycle codes are useful only for loop configurations, where the probe power at the input of the sensing fiber has a reduced power.

\section{EXPERIMENTAL RESULTS}

We assembled a BOTDA setup following the scheme in Fig. 2 to demonstrate the proposed technique. The optical source was a DFB laser at $1550 \mathrm{~nm}$, whose output was divided by a coupler into two branches. In the upper branch, the pump signal was pulsed using a semiconductor optical amplifier (SOA). Then, the pulse power was amplified to a peak of $20 \mathrm{dBm}$ in an EDFA, and the polarization state of the pulsed pump was randomized with a polarization scrambler before being launched into the sensing fiber.

In the lower branch of the setup, a dual-probe wave was generated using a Mach-Zehnder electrooptic modulator (MZ-EOM). This modulator was driven by an arbitrary waveform generator (AWG), and biased for minimum transmission so as to generate a FM-modulated double-sideband suppressed-carrier signal. For this purpose, the 


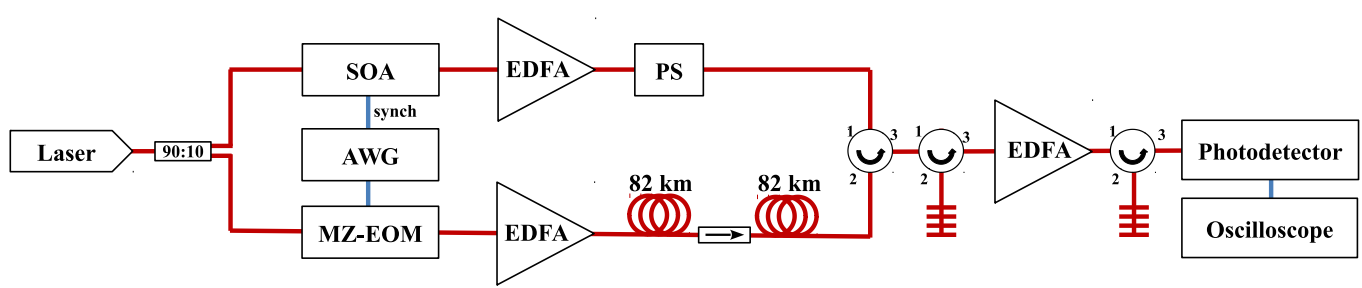

Figure 2: Experimental setup for the BOTDA sensor.

AWG provides a microwave signal with an instantaneous frequency varying around the Brillouin frequency shift (BFS) of the fiber following a saw-tooth shape with a period of $10.7 \mu$ s and peak-to-peak frequency deviation of $250 \mathrm{MHz}$. Then, the signal was boosted to $15 \mathrm{dBm}$ by another EDFA before injecting it in the 82-km leading fiber. At the input of the $82 \mathrm{~km}$ of fiber-under-test (FUT) the probe power was $-1.8 \mathrm{dBm}$. Finally, before the receiver, two tunable narrowband fiber Bragg gratings (FBG) were used to filter out one of the probe sidebands and the Rayleigh backscattering originating from the pump wave. An EDFA was used between the two FBGs as a pre-amplifier.

To analyze the performance of the sensor in terms of measurement precision, a series of 10 consecutive measurements for stable temperature conditions were performed over a loop of $164 \mathrm{~km}$ length of standard singlemode fiber. The pulse duration was set to $10 \mathrm{~ns}$, corresponding to approximately $1-\mathrm{m}$ spatial resolution, and the code length was set to $\mathrm{L}=79$. The final $5 \mathrm{~km}$ of the fiber was placed loose in a climatic chamber, while the rest was held at room temperature in a spool. The frequency step of the spectral scan was $2.3 \mathrm{MHz}$ and 16000 averages were used to obtain the final traces. From these measurements, the uncertainty of the BFS measurement, which is depicted in Fig. 3a, was found to be $3 \mathrm{MHz}(1 \sigma)$ at the end of the fiber under test. Fig. 3b portrays the BFS along the whole sensing fiber, where four fiber spools are clearly distinguishable, with a fairly uniform BFS of all of them. The BFS along the fiber was calculated by performing a quadratic fit on the Brillouin gain spectrum (BGS). The noise reduction effect provided by the coding technique can be clearly appreciated in Fig. 3c, which compares the conventional BOTDA trace with that obtained with coding. The measured SNR enhancement was $6.2 \mathrm{~dB}$, in good agreement with the theoretical expected value $(6.5 \mathrm{~dB})$ in case of a 79-bit code length.

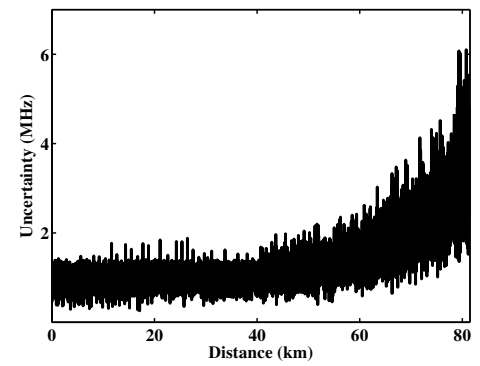

(a)

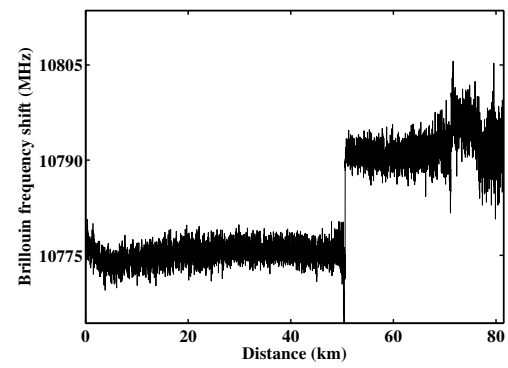

(b)

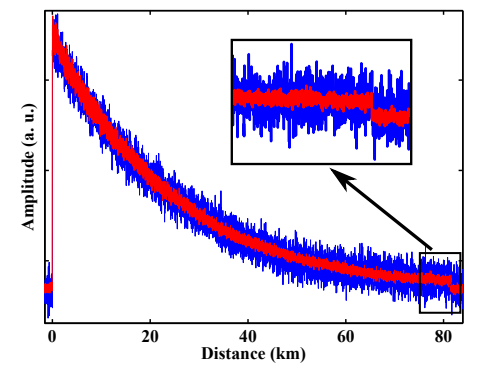

(c)

Figure 3: (a) BFS uncertainty ( $1 \sigma$ ) obtained by the technique (b) Distribution of the BFS profile and (c) BOTDA traces at $10.775 \mathrm{GHz}$ obtained for the analyzer with a 79-bit cyclic coding (red) and without aplying coding (blue).

We also studied the limitations on the probe and pump power that can be injected in the fiber using monocolor cyclic coding in order to avoid distortion of the decoded signal. For this purpose, we replaced the $164 \mathrm{~km}$ fiber spools by a $25 \mathrm{~km}$ length of standard single-mode fiber. The probe power was fixed to $-4 \mathrm{dBm}$ to ensure that non-local effects are avoided, and the pump wave was modified in terms of pulse duration $(20 \mathrm{~ns}, 30 \mathrm{~ns}$ and $40 \mathrm{~ns}$ ), pump power and code length. Fig. 4a highlights the deleterious effect on the decoded signal due to the non-linear amplification as a function of the code length for a pulse of $20 \mathrm{~ns}$ and $19 \mathrm{dBm}$ power. The 
non-linear amplification due to the code length, as can be clearly observed in Fig. 4b, where it is depicted the amplitude value of the beginning of the decoded BOTDA trace at maximum BFS frequency for different code lengths, has a direct link to the pump power, the code length and also to the pump duration. Thus, in order to avoid distortions in the decoded signal, it is mandatory to reduce the pump power, the code length or the pump duration, which can lead, in several cases, to a non-existing improvement of the SNR.

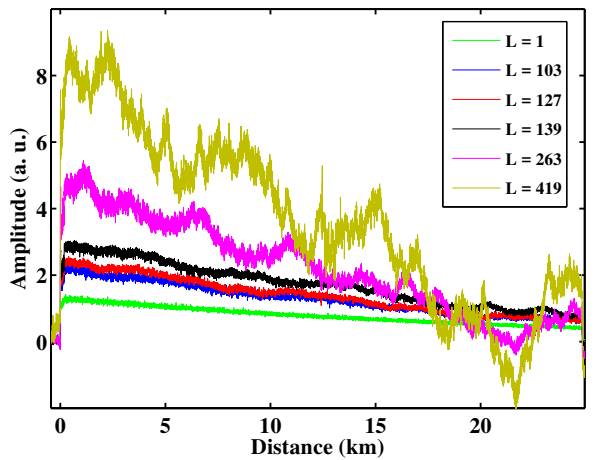

(a)

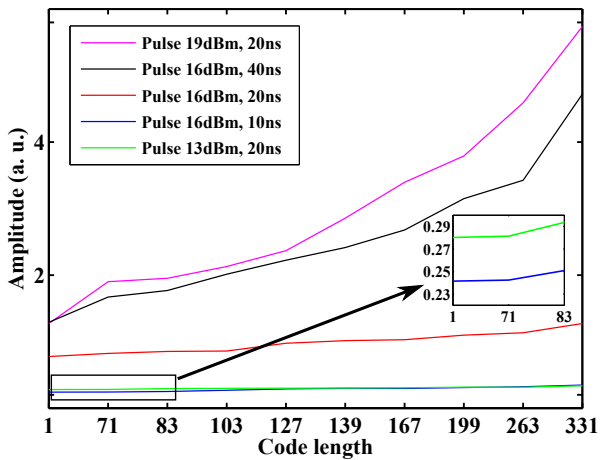

(b)

Figure 4: (a) Obtained BOTDA traces at maximum BFS frequency for a pump of $19 \mathrm{dBm}$ and different code lengths L (b) Non-linear amplification for different code lengths, and different pulse power and duration.

\section{CONCLUSION}

In this paper, we have introduced an enhanced BOTDA that combines mono-color cyclic coding and a frequency modulated dual-probe. The capabilities of the technique have been analyzed by performing measurements over a $164 \mathrm{~km}$ loop length of fiber with high precision and resolution. Furthermore, a brief study regarding the limits of the code lengths that can be used without distortion in the decoded signal has been presented.

\section{ACKNOWLEDGMENTS}

The authors wish to acknowledge the financial support of the Spanish Ministerio de Economía y Competitividad through the projects TEC2013-47264- C2-2-R and TEC2016-76021-C2-1-R, FEDER funds and the UPNA.

\section{REFERENCES}

[1] Thévenaz, L., Mafang, S. F., and Lin, J., "Effect of pulse depletion in a brillouin optical time-domain analysis system," Opt. Express 21, 14017-14035 (Jun 2013).

[2] Dominguez-Lopez, A., Angulo-Vinuesa, X., Lopez-Gil, A., Martin-Lopez, S., and Gonzalez-Herraez, M., "Non-local effects in dual-probe-sideband brillouin optical time domain analysis," Opt. Express 23, 1034110352 (Apr 2015).

[3] Shimizu, T., Nakajima, K., Shiraki, K., Ieda, K., and Sankawa, I., "Evaluation methods and requirements for the stimulated brillouin scattering threshold in a single-mode fiber," Opt. Fiber Tech. 14(1), 10 - 15 (2008).

[4] Floch, S. L., Sauser, F., Llera, M., and Rochat, E., "Novel brillouin optical time-domain analyzer for extreme sensing range using high-power flat frequency-coded pump pulses," Journal of Lightwave Technology 33, 2623-2627 (June 2015).

[5] Le Floch, S., Sauser, F., Llera, M., and Rochat, E., "Colour cyclic code for brillouin distributed sensors," Proc. SPIE 9634, 963431-963431-4 (2015).

[6] Mompó, J. J., Urricelqui, J., and Loayssa, A., "Brillouin optical time-domain analysis sensor with pump pulse amplification," Opt. Express 24, 12672-12681 (Jun 2016).

[7] Ruiz-Lombera, R., Urricelqui, J., Sagues, M., Mirapeix, J., López-Higuera, J. M., and Loayssa, A., "Overcoming nonlocal effects and brillouin threshold limitations in brillouin optical time-domain sensors," IEEE Photonics Journal 7, 1-9 (Dec 2015). 